\title{
HERVs, immunity, and autoimmunity: Understanding the connection
}

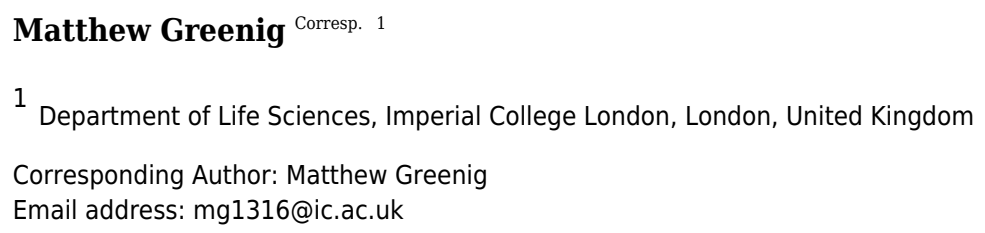

Since their discovery in the 1960s, further investigation into human endogenous retroviruses (HERVs) has challenged the conventional view of viral sequences as exclusively parasitic elements. Once presumed to be a group of passive genetic relics, it is becoming increasingly clear that this view of ERVs, while generally accurate, is incorrect in many specific cases. Research has identified ERV genes that appear to be co-opted by their mammalian hosts, but the biological function of ERV elements in humans remains a controversial subject. One area that has attracted some attention in this domain is the role of co-opted ERV elements in mammalian immune systems. The relationship between ERVs and human autoimmune diseases has also been investigated, but has historically been treated as a separate topic. This review will summarize the current evidence concerning the phenotypic significance of ERVs, both in the healthy immune system and in manifestations of autoimmunity. Furthermore, it will evaluate the relationship between these fields of study, and propose previously-unexplored molecular mechanisms through which HERVs might contribute to certain autoimmune pathologies. Investigation into these novel mechanisms could further our understanding of the molecular basis of autoimmune disease, and may one day provide new targets for treatment. 


\section{HERVs, immunity, and autoimmunity: Understanding} 2 the connection

3

4

5

6

7

8 Corresponding Author:

9 Matthew Greenig 1

10108 Verdun Road, London, UK, SW13 9AN

11 Email address: mg1316@ic.ac.uk

12

13

14

15

16

17

18

19

20

21

22

23

24

25

26

27

28

29

30

31

32

33

34

35

36 


\section{Abstract}

38

39

54

55

56

57

Since their discovery in the 1960s, further investigation into endogenous retroviruses (ERVs) has challenged the conventional view of viral sequences as exclusively parasitic elements. Once presumed to be a group of passive genetic relics, it is becoming increasingly clear that this view of ERVs, while generally accurate, is incorrect in many specific cases. Research has identified ERV genes that appear to be co-opted by their mammalian hosts, but the biological function of ERV elements in humans remains a controversial subject. One area that has attracted some attention in this domain is the role of co-opted ERV elements in mammalian immune systems. The relationship between ERVs and human autoimmune diseases has also been investigated, but has historically been treated as a separate topic. This review will summarize the current evidence concerning the phenotypic significance of ERVs, both in the healthy immune system and in manifestations of autoimmunity. Furthermore, it will evaluate the relationship between these fields of study, and propose previously-unexplored molecular mechanisms through which HERVs might contribute to certain autoimmune pathologies. Investigation into these novel mechanisms could further our understanding of the molecular basis of autoimmune disease, and may one day provide new targets for treatment.

\section{Introduction}

It has long been known that up to $8 \%$ of the human genome is derived from a large number of viral elements (International Human Genome Sequencing Consortium, 2001; Patel et al., 2012). These endogenous viral elements (EVEs) are present in modern humans as the result of ancient viral integration events into the germline cells of our ancestors. The process of viral germline integration is known as endogenization, and has likely occurred many times throughout 
63 mammalian evolution, with certain EVEs estimated to have integrated over 100 million years

64 ago (Lee et al., 2013). Investigation into these ancient viral elements has given rise to a new field

65 of study known as paleovirology, an area that focuses particularly on the evolutionary history

66 and present-day biological significance of EVEs.

67

68 Predictably, the majority of EVEs appear to be derived from retroviruses (Katzourakis and

69 Gifford, 2010), the only animal viruses that undergo genomic integration as part of their normal

70 life cycle. Exogenous retroviruses are transmitted as $(+)$ strand RNA, packaged in a protein

71 capsid and lipid envelope. Upon entering the cell, they undergo a unique step in their life cycle

72 that involves reverse transcription of their single-stranded RNA to form double-stranded DNA

73 (dsDNA). The virus is then transported to the nucleus, where it mediates integration of the

74 newly-formed viral dsDNA into the host's genome. This mechanism is displayed in fig. 1.

75 Retroviral endogenization mechanisms are well-characterized, and currently, ERVs constitute

76 the most thoroughly investigated subset of EVEs.

77

78 Exogenous viruses are transmitted horizontally, moving between individuals whose cells provide

79 the machinery required for the virus to replicate itself and release infectious particles, so that

80 other susceptible individuals can be infected. Retroviruses can be transmitted in this way, but

81 their permanent integration into the host cell's genome also allows for another mode of

82 transmission to occur. If a retrovirus integrates into the genome of a germline cell that

83 contributes to the formation of a nascent individual, the integrated provirus will be transferred

84 from parent to offspring - a process known as vertical transmission. If retroviral integration

85 occurs before the first cell division of a zygote, all of the progeny's somatic cells, and at least 
86 half of their gametes, will contain the integrated retrovirus. The retroviral DNA is then present in

87 and can be transferred through the organisms's germline; the virus has become endogenized.

88 Immediately after endogenization, most new ERVs probably remain replication-competent, so

89 viral re-integration events following endogenization are still possible; these would increase

90 provirus copy number in the host and could increase the ERV's frequency in the gamete

91 population, if the virus was transmitted between gametes. Indeed, some work has identified

92 human ERV (HERV) families with particularly high copy numbers (200+ copies) (Belshaw et

93 al., 2005). Yet, although we have identified actively-expressed ERVs in many other vertebrates

94 (Aswad et al., 2012), nearly all human ERVs discovered thus far have accumulated mutations

95 rendering them replication-incompetent, and their protein products defective (Weiss, 2016).

96 Most HERVs are not even actively transcribed (Oja et al., 2007). However, these generalizations

97 do not apply to the entire group.

98

99 Some of the earliest evidence that certain HERVs might be expressed in modern humans came

100 from the discoveries of Syncytins 1 and 2, two proteins that are expressed in developing human

101 embryos. These proteins are full-length products derived from integrated retroviral envelope

102 genes (env); they mediate fusion between placental cells, a process that is disrupted in their

103 absence (Sha et al., 2000). As some of the earliest discovered examples of co-opted retroviral

104 elements, syncytins have prompted further research into host domestication of endogenous

105 retroviral genes. Later bioinformatics-based investigations revealed that a small but noteworthy

106 proportion of ERVs in the human genome contain ORFs encoding for intact viral polyproteins

107 (Villesen et al., 2004). The traditional view of ERVs assumes them to be defunct copies of

108 ancient exogenous retroviruses, whose sequences have persisted in the human germline precisely 
109 because of accumulated mutations that prevent the virus from replicating, converting the ERV

110 into a neutral locus. Neutral loci are expected to undergo mutational decay over large periods of

111 time, so the presence of intact HERV ORFs in the human genome could be an indicator of

112 relatively recent endogenization events. However, an alternative explanation suggests that these

113 sequences have been selectively maintained throughout evolutionary history, after being co-

114 opted for some beneficial function. These hypotheses are not mutually exclusive, and both would 115 imply that certain HERV elements might still be actively expressed in modern day humans. One

116 microarray-based assay observed HERV transcription in every sample of 19 different human

117 tissue types (Seifarth et al., 2005), indicating that HERV expression might be relatively

118 ubiquitous throughout the human body.

120 Taken together, these results suggest that the view of HERVs as inactive, neutral sequences does 121 not apply to all endogenous retroviral elements in the human genome. It is well documented that 122 ERVs are not only expressed, but produce phenotypic consequences in other vertebrates (Aswad 123 and Katzourakis, 2012). Investigation into the function of co-opted ERVs in the immune systems 124 of various vertebrate species has revealed complex, intimate relationships between endogenous 125 retroviruses and their hosts (Aswad and Katzourakis, 2012; Frank and Feschotte, 2017). Despite 126 the ubiquity of such relationships, the phenotypic effects of HERV elements on the immune 127 systems of their human hosts largely remain a mystery. Accordingly, much controversy has 128 emerged regarding the link between HERVs and human autoimmune diseases. Increased HERV 129 activity has been identified as a key feature in various forms of autoimmunity (Bjørn et al., 130 2016), and while mechanistic theories have been formulated to explain their contribution to 131 disease pathogenesis (Balada et al., 2009), there is still no consensus regarding the connection 
132 between HERVs and autoimmunity. Furthermore, the relationship between HERV function in

133 the healthy immune system and dysfunction in autoimmune disease remains mostly unexplored.

134 In the present paper, I review some of the existing evidence on interactions between endogenous

135 retroviruses and mammalian immune systems, both in the context of normal immunity and

136 autoimmunity. In addition, I utilize a unique cross-disciplinary framework for evaluating the

137 relationship between HERV elements and human autoimmune diseases, incorporating principles

138 from retrovirology, immunology, and our knowledge of ERV/host interplay in vertebrate

139 immune systems. Using this cross-disciplinary approach, I formulate and present multiple novel

140 hypotheses concerning HERV-mediated mechanisms of autoimmunity.

141

142

143

144

145 This review discusses a subject that exists at the interface between evolutionary biology,

146

147 including those dedicated to specific research in either virology or immunology, as well as work

148

149

150

151

152

153

154

155

156

\section{Survey methodology} virology, and immunology. Therefore, sources from a wide range of journals were used, published in journals with broader research themes, e.g. Nature. The vast majority of citations derive from primary research, and quantitative evidence is cited whenever available.

EVE-Derived Immunity. Host-virus interactions over large evolutionary time scales are generally described using an 'arms race' model, in which the virus and host 'race' to evolve novelties that allow them to successfully outcompete one another. As one might expect, this evolutionary competition has driven the evolution of animal immune system genes to a huge extent (Daugherty and Malik, 2012). In the prototypical model for natural selection, evolutionary novelties develop through mutation of pre-existing genes. Viruses clearly have the edge in this 
157 regard, boasting higher mutation rates, faster generation times, and an astounding ability to

158 handle mutational load (Lauring et al., 2013). However, co-option of an endogenous viral gene

159 provides a powerful weapon for their competing animal hosts, introducing an entirely novel

160 element to the host-virus arms race, to which a competing virus has not developed resistance.

161 Therefore, although retroviral integration can lead to disease, insertional oncogenesis, or

162 disruption of host genes, co-opted ERVs can also offer a crucial edge to their hosts in the

163 molecular arms race. This advantage might explain the observed ubiquity of ERV-encoded

164 products in vertebrate immune systems (Aswad and Katzourakis, 2012; Frank and Feschotte,

165 2017), despite the improbability of ERV co-option events and the fitness costs associated with 166 retroviral endogenization events.

167

168 Co-opted ERVs can be broadly divided into two categories: elements whose function derives

169 from their encoded product(s), and elements that act as regulatory sequences in the host genome.

170 Examples of both appear in mammalian immune systems (see fig. 1).

172 While recent investigation has suggested that some EVEs confer antiviral immunity through non173 coding RNAs (Honda and Tomonaga, 2016), most research to date regarding ERV products has

174 focused on ERV-encoded proteins. Restriction factors are a class of cellular proteins that actively 175 inhibit viral replication (Goff, 2004), and certain co-opted ERVs have been identified to act as 176 restriction factors against exogenous retroviruses. So far, no human ERVs with these properties 177 have been discovered (Frank and Feschotte, 2017), but other research has identified a number of 178 ERV-derived restriction factors in the murine model, as well as in sheep, chickens, and cats 179 (Aswad and Katzourakis, 2012). 
180

181

182 183

184

185

186

187

188

189

190

191

192

193

194

195

196

197

198

199

200

201

202

203

Co-opted ERVs in the Immune System. Fv-4 is one ERV-derived restriction factor that has been identified in mice. The Fv-4 locus contains a fully intact env gene with $>70 \%$ sequence similarity to env in multiple strains of exogenous murine leukemia virus (MuLV) (Ikeda et al., 1985). Fv-4 expression was later demonstrated to directly confer resistance to ecotropic MuLV in transgenic mice; host resistance to the virus also correlated with the levels of Fv-4 expressed (Limjoco et al., 1993). Further work has been done to elucidate the ERV-encoded protein's mechanism of action. While viral Env proteins usually bind to receptors on the cell surface to mediate cell entry, exogenous MuLV Env has been shown to be capable of interacting with its receptors intracellularly in the endoplasmic reticulum, as demonstrated by pulse-chase labelling (Kim and Cunningham, 1993). Kim and colleagues observed that this intracellular binding interfered with receptor maturation by inhibiting N-linked glycosylation of two residues, and that receptor binding to exogenous MuLV was significantly reduced in cells expressing MuLV Env. However, they also observed that mutant non-glycosylated forms of the receptor bind extracellular MuLV at the same efficiency as the wild type receptor, in the absence of intracellular MuLV Env. Thus, the ability of intracellular MuLV Env to inhibit MuLV infection could rely on intracellular retention of the MuLV receptor due to ligand binding, or, on competitive inhibition of virus binding sites, reducing the number of receptors available to exogenous MuLV on the cell surface. Figure 2 provides a general schematic for these resistance mechanisms. Later work also revealed that one of the amino acid residues at which Fv-4 differs from exogenous MuLV Env is necessary for the Env protein's fusogenic capabilities; exogenous Env that is modified with the Fv-4 mutation at this residue is unable to fuse with target cell membranes, and forms uninfectious virion particles (Taylor et al., 2001). Due to its homology to exogenous MuLV Env, Fv-4 can likely be incorporated into MuLV virions, thereby producing 
204 uninfectious particles with non-fusogenic Env proteins. Thus, although its restriction mechanism

205 has not been confirmed, Fv-4's antiviral activity can likely be attributed to its homology to

206 MuLV Env. This example illustrates the broader point that certain ERVs have maintained high

207 levels of structural similarity to their exogenous counterparts throughout evolutionary history,

208 which could allow them to interact with host proteins that normally interact with exogenous

209 viruses.

210

211 Non-Env restriction factors derived from ERVs have also been identified in the murine model.

$212 \mathrm{Fv}-1$ is one such protein, first observed in the 1970s to confer resistance to MuLV (Pincus et al.,

213 1970). Fv-1 appears to be derived from a retroviral gag gene (Best et al., 1996), the domain

214 responsible for encoding viral capsid elements. Fv-1 appears to restrict MuLV by a mechanism

215 similar to that of the TRIM5 $\alpha$ restriction factor expressed in humans, which also restricts MuLV

216 (Yap et al., 2004). TRIM5 $\alpha$ is known to restrict retroviral infection through interaction with the

217 viral capsid (Sastri and Campbell, 2011), and restriction by both Fv-1 and TRIM5 $\alpha$ relies on

218 specificity at the same single amino acid in MuLV's capsid protein, suggesting a common

219 mechanism of action (Kozak and Chakraborti, 1996; Yap et al., 2004). In addition, phylogenetic

220 analysis of $\mathrm{Fv}-1$ genes in different mouse subgenera has provided evidence of positive selection

221 in different variants of Fv-1 (Yan et al., 2008). Specifically, dN/dS $>1$ was noted at three codons

222 in a segment known to be involved in capsid interactions, indicating an evolutionary history of

223 host-virus interactions at this site. Being derived from a retroviral gag gene, Fv-1's homology to

224 retroviral capsid elements likely enables it to bind exogenous viral capsids, which themselves are

225 assembled through high-affinity interactions between structurally identical monomeric subunits. 
226 Importantly, both Fv-4 and Fv-1 illustrate a broader theme of molecular mimicry in co-opted

227 ERV elements: products with homology to components of exogenous viruses.

228

229 A separate area of research has investigated the role of co-opted HERVs as regulatory sequences, 230 specifically retroviral long terminal repeat domains (LTRs). In exogenous retroviruses, LTRs 231 function primarily as cis-regulatory sequences for viral genes (Temin et al., 1981). However, 232 multiple examples of LTR co-option in mammals have been discovered in recent years (Franke 233 et al., 2017). These findings extend to immunology, where co-opted LTRs have been implicated 234 in the human interferon (IFN) response (Chuong et al., 2016). Chuong and colleagues analysed 235 the role of ERV LTRs in promoting the transcription of IFN- $\gamma$-stimulated genes. Their findings 236 revealed that certain ERV LTRs are bound by STAT1 and/or IRF1, transcription factors that 237 regulate interferon-induced genes. These LTRs were strongly enriched near interferon-stimulated 238 sequences, suggesting a possible role for them in IFN- $\gamma$ gene regulation. Chuong's group further 239 investigated MER41, a family of HERVs shown to be a source of IFN-inducible binding sites.

240 An LTR belonging to the MER41 family (MER41.AIM2) was shown to be the only STAT1

241 binding site within 50kb of the IFN- $\gamma$-inducible gene Absent in Melanoma 2 (AIM2) gene, a

242 foreign DNA sensor involved in the innate immune response. MER41.AIM2 thus appears to be a 243 primary STAT1 promoter for AIM2, and CRISPR-Cas9 deletions in HeLa cell lines 244 demonstrated that MER41.AIM2 knockout rendered cells unable to express AIM2 upon IFN- $\gamma$ 245 treatment (Chuong et al., 2016), as shown in figure 3.

247 Chuong and colleagues (2016) also found MER41 LTR elements within 20kb of three other 248 interferon-inducible genes, and genetic knockouts showed that each of these ERV promoters 
249 contributed to expression of IFN- $\gamma$-induced genes. Interestingly, it was discovered separately that 250 exogenous HIV-1 LTRs contain an IRF1 binding site (Sgarbanti et al., 2008), a feature that 251 likely evolved as a way for the virus to regulate its expression in response to host immune 252 signalling. When considered with $M E R 41$ 's role in immunoregulation, these results imply that 253 the evolution of host transcription factor binding sites in exogenous viral sequences might 254 actually facilitate co-option of ERV regulatory elements. Exogenous retroviruses that contain 255 regulatory sequences with such binding sites might not require significant changes in nucleotide sequence to be used as host promoters, as they interact with host transcription factors in their exogenous form. Furthermore, because many retroviruses have evolved to infect immune system cells (Coffin et al., 1997), systems of retroviral gene regulation that operate through interaction with host transcription factors might specifically target molecular components of the immune response, facilitating their co-option in the immune system specifically.

HERVs and autoimmune disease. Autoimmune diseases (AD) are complex pathologies defined by a breakdown of immunological tolerance to self-molecules, and the resulting immune responses mounted against those components of the body (Smith and Germolec, 1999). However, some of these aberrant responses (and their associated diseases) are not well-defined on physiologically, raising the possibility that some autoimmune diseases are actually multiple phenotypically distinct conditions with similar manifestations. Therefore, in attempting to understand their etiology, it must be noted that some causative mechanisms of disease pathogenesis might apply to some, but not all of the entire patient population. While genetic background seems to affect incidence rates of $\mathrm{AD}$, it is not sufficient for the development of

271 most forms of autoimmunity (Meda et al., 2011). To quantify the significance of genetic factors 272 in the development of a given disease, monozygotic twin concordance rates (rates of disease co- 
273 incidence) can be measured. Incomplete concordance is observed in many autoimmune diseases

274 (Bogdanos et al., 2012), implicating a mix of genetic and environmental factors in their etiology.

275

276 The etiology of multiple sclerosis (MS), for example, appears to be associated with components

277 on multiple levels. Multiple sclerosis is an autoimmune disease that affects millions of people,

278 characterized by inflammation of the myelin sheaths that coat nerve axons (Stüve and

279 Oskenberg, 2006). Early studies on twin concordance rates in a North American population

280 revealed a partial genetic association for the disease (Islam et al., 2006). Using a different

281 approach, genome-wide association studies have identified SNPs that correlate with MS

282 incidence (Alcina et al., 2012). Epigenetics are also thought to be involved in the development of

283 MS (Küçükali et al., 2015), yet the specific mechanisms by which they contribute remain

284 unclear. A number of environmental factors have been identified to correlate with disease

285 development, including vitamin D, smoking, and Epstein-Barr Virus (EBV) (Gorman et al., 286 2012).

287

288 HERVs appear to have a significant connection with certain autoimmune diseases, but much

289 controversy has surrounded their exact relationship, whether as innocuous by-products, indirect

290 contributors, or directly causative agents of disease symptoms (Balada et al., 2010). Regardless

291 of their exact contribution to disease pathogenesis, a large body of evidence regarding HERV

292 association with AD, consisting of multiple unrelated observations in distinct pathologies, has

293 accumulated over the years. Some of the earliest evidence for HERV association with certain AD

294 emerged through the observation of increased HERV RNA expression through techniques like

295 RT-PCR (Garson et al., 1998; Ogasawara et al., 2001). Other work has identified anti-HERV 
296 antibodies in the blood of patients afflicted with rheumatic autoimmune disease (Hervé et al.,

297 2001). More recently, HERV protein production has been compared in healthy individuals and

298 MS patients, revealing certain HERV products that appear to be upregulated in disease (Laska et

299 al., 2012). However, despite extensive evidence of correlation, limited progress has been made in 300 determining the specific contributions of HERVs to the induction of autoimmunity.

301

302 Rheumatoid Arthritis. Nevertheless, large strides have been made in improving our 303 mechanistic understanding of HERV involvement in certain autoimmune diseases. The most 304 prevalent of these is rheumatoid arthritis (RA), an autoimmune condition that affects 305 approximately 5 in 1000 people (Aletaha and Smollen, 2018). The main pathological feature of 306 RA is inflammation of synovial joints, which is mediated by a variety of immune system cells 307 and molecules (Guo et al., 2018). Autoantibodies appear to play a key role in disease 308 progression, but large variation exists in the specific autoantibody epitope profiles observed in 309 the sera of infected patients (Aletaha and Blüml, 2016). Our current understanding of HERV 310 involvement in RA pathogenesis is supported by a large body of evidence, and therefore the 311 disease will be used here as an illustrative example, to demonstrate how mechanistic hypotheses 312 of HERV contributions to autoimmune disease are formulated and tested.

314 A landmark experiment in the early 2000s was the first to establish a strong connection between 315 human endogenous retroviruses and RA incidence (Hervé et al., 2001). Hervé and colleagues 316 compared sera from RA patients to healthy controls and demonstrated that a significantly greater 317 proportion of RA patients were seropositive for antibodies specific to envelope proteins from 318 two members of the HERV-K family: HERV-K10 and IDDMK $_{1,2} 22$. Importantly, their group 
319 also mapped the epitopes of anti-HERV-K antibodies extracted from diseased patients, and

320 identified multiple distinct reactive peptide epitopes, all arising from HERV-K sequences. These

321 results implied that the antibodies detected by their group were, in fact, driven by a specific

322 response to HERV-K antigens. Later work used RT-qPCR to more precisely quantify expression 323 of HERV-K10 gag mRNA in peripheral blood mononuclear cells (PBMCs) extracted from RA

324 patients, revealing significantly higher expression levels in rheumatoid patients compared with 325 both disease controls and healthy controls (Ejtehadi et al., 2006). The group also noted that 326 HERV-K10 gag expression was increased almost two-fold at sites of active inflammation, as 327 opposed to the peripheral blood.

328

329 More evidence of HERV involvement was presented in a 2010 study that used bioinformatics 330 analysis to map sequence alignments between HERV and human autoantigens, in order to 331 identify potential cross-reactive epitopes (Freimanis et al., 2010). Their findings revealed 332 multiple pairs of HERV and host proteins with shared epitopes, including one specifically cross333 reactive pair in HERV-K10 Gag1 protein and human type II collagen. Type II collagen 334 autoantibodies were known to be associated with RA (Choi et al., 1988), and so Freimanis and 335 colleagues hypothesized that anti-HERV-K Gag1 antibodies, which had previously been 336 observed in RA patients (Hervé et al., 2001), could be cross-reacting with human collagen in 337 vivo and contributing to disease pathogenesis. Crucially, this hypothesis predicted that their 338 group could expect to find, in the sera of RA patients, autoantibodies cognate to the specific 339 HERV-K10 Gag1 epitope identified in their bioinformatics analysis. Indeed, they performed 340 ELISA assays to quantify the levels of antibodies specific to this epitope present in the sera of 341 RA patients, and observed titers significantly higher than all controls, including multiple non-RA 
342 disease control groups. Taken together with previous evidence of HERV-K antigen-driven

343 autoantibody production (Hervé et al., 2001) and increased HERV-K10 gag expression levels in

344 RA (Ejtehadi et al., 2006), the results obtained by Freimanis et al. (2010) suggest a pathological

345 role for HERV-K10 gag sequences in disease. Specifically, their findings indicate that an

346 autoantibody response against a HERV-K Gag1 epitope that cross-reacts with type II collagen

347 may contribute to certain manifestations of RA, targeting immune system effector functions to 348 synovial joints.

349

350 Subsequent findings affirmed the correlation between anti-HERV-K Gag1 antibodies and

351 rheumatoid arthritis, but failed to identify a correlation between serum antibody concentrations

352 and disease severity (Nelson et al., 2014). However, it should be noted that Nelson's group used

353 a Gag1 epitope different to the type II collagen cross-reactive sequence identified by Freimanis

354 et al. (2010). There is ongoing debate about which HERV-K sequences are the most likely to

355 induce the production of cross-reactive antibodies in RA. Thus, while some evidence supports a

356 pathological role for HERVs in disease, such theories must be substantiated by observations of

357 specific correlations between HERV-K activity and disease progression. The works highlighted

358 in this section are not used to present an argument that HERVs act as etiological agents in RA.

359 Rather, they provide a subject-specific example to illustrate how predictive, mechanistic

360 hypotheses in investigations of disease etiology can be formulated from existing evidence. In the

361 remainder of this review, I use the available evidence to construct novel hypotheses regarding

362 HERV involvement in two diseases that are highly-associated with HERVs, but less well-

363 understood: Multiple Sclerosis and Lupus. 
365 Multiple Sclerosis. Multiple sclerosis is a well-studied autoimmune disease that exhibits a 366 strong correlation with expression of multiple HERV families (Morandi et al., 2017). Despite the 367 disease's prevalence, and substantial evidence of correlations between HERV expression and 368 disease incidence, our mechanistic understanding of HERV involvement in MS has lagged 369 behind our understanding of their involvement in RA. Determining the underlying reasons for 370 the increased HERV expression observed in MS could improve our understanding of their 371 connection with the disease, and with autoimmunity in general. EBV is one interesting factor to 372 consider. The virus has not only been detected in the sera of 100\% of MS patients (Pakpoor et 373 al., 2012); it also appears to activate expression of certain HERV elements in both healthy and 374 MS cell lines (Mameli et al., 2012). The tripartite relationship between HERVs, EBV, and MS 375 has received some attention in immunological research, and there is substantial evidence of 376 EBV's ability to induce increased HERV expression in cells from MS patients, both in vitro 377 (Mameli et al., 2012) and in vivo (Mameli et al., 2013). Other viruses like HIV and Herpes 378 Simplex Virus have also been demonstrated to interact with endogenous retroviral sequences 379 (Lee et al., 2003; Bhardwaj et al., 2014). It is well-established that infections can contribute to 380 the development of various autoimmune diseases (Ercolini and Miller, 2009), and these findings 381 may provide a link between autoimmunity triggered by exogenous viral infection, and the 382 increased HERV expression observed in many AD. In fact, in light of strong evidence that some 383 HERV-derived promoter regions are regulated by immune system signalling molecules

384 (Manghera and Douville, 2013; Chuong et al., 2016), it is possible that transcription factors like 385 STAT1 - whether induced by infection, or another factor - could be sufficient to activate certain 386 HERV regulatory elements and their downstream genes. 
388 Using a different approach to investigate HERVs in MS, genome-wide association studies have 389 recently attempted to characterize distinct HERV variants that correlate with disease. It was 390 discovered that certain SNPs in sequences of the HERV-K family exhibit significant correlations 391 with MS incidence (Nexø et al., 2015). Some of the risk alleles identified by Nexø and 392 colleagues were SNPs located upstream of their adjacent HERV-K genes, suggesting that certain 393 promoter alleles might induce increased expression of their downstream viral elements in MS 394 patients. These findings provide strong evidence of HERV-K contribution to MS, but genome395 wide association studies are not always suitable for identifying complex, multifactorial 396 mechanisms of disease induction. Autoimmune diseases are particularly problematic, being 397 defined in most cases by organism-level immune system interactions that can likely be induced 398 by multiple distinct molecular mechanisms. Even assuming a strong genetic basis for disease, 399 specific variants at multiple distinct HERV loci might be capable of producing identical 400 pathological effects, further reducing association between any individual gene and disease 401 incidence. The extent of this problem is realized when one considers the vast number of ERV 402 sequences in the human genome and the fact that many of them exist at large copy numbers with 403 high levels of sequence similarity, each group having arisen from replication of a single 404 405 endogenized retrovirus (Belshaw et al., 2005). Therefore, understanding the etiology of autoimmune diseases (especially with regards to HERVs) requires the formulation of specific, 406 testable, and mechanistic hypotheses of pathogenesis. Such hypotheses can be tested in animal 407 models with genetic knockouts or modifications of proposed components, so that disease 408 manifestations can be evaluated on a whole-organism level and theories can be rigorously 409 evaluated. Here, I present one potential mechanism through which HERV elements might 410 contribute to the manifestation of MS. Many other theories have also been proposed (Perron et 
411 al., 2001; Balada et al., 2009; Hummel et al., 2015; Nexø, 2018), and it should be noted that

412 most of them are not mutually exclusive.

413

414 Many HERV proteins exhibit sequence homology to proteins produced by exogenous

415 retroviruses. As has been demonstrated in mice, this structural similarity can allow ERV-encoded

416 products to serve as antiviral restriction factors (Pincus et al., 1970; Limjoco et al., 1993).

417 However, HERV molecular mimicry might also be capable triggering antiviral responses in the

418 absence of exogenous viral infection. There is evidence to suggest that HERV-encoded products

419 could contribute to the activation of dendritic cells (DCs) in MS, an important factor in the

420 initiation of disease that leads to various downstream effects that produce inflammatory

421 symptoms (Grigoriadis and van Pesch, 2015). One possible mediator for this interaction is

422 TRIM5 $\alpha$. As previously mentioned, TRIM5 $\alpha$ is a retroviral restriction factor that inhibits viral

423 replication through interaction with the viral capsid (Sastri and Campbell, 2011). In addition to

424 its direct antiviral activity, TRIM5 $\alpha$ also contributes to innate immune signalling in dendritic

425 cells, through K63-linked ubiquitination and activation of the TAK1 kinase complex, which

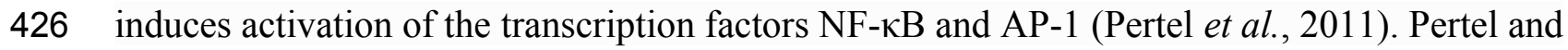

427 colleagues showed that the effect of TRIM5 $\alpha$-mediated signalling was highly specific, with

428 TRIM5 $\alpha$ knockdown only significantly decreasing expression of 33 genes, about $70 \%$ of which

429 encoded pro-inflammatory cytokines. Crucially, they also demonstrated that production of

430 inflammatory cytokines in DCs was upregulated in response to increased TRIM5 $\alpha$ interaction

431 with retroviral capsid lattices. There is evidence that miscalibration of these signalling pathways

432 could play a role in the induction of MS. 
434 Constitutive TRIM5 $\alpha$ activity provides a plausible explanation for the aberrant DC activation 435 observed in MS. One large genome-wide association study with over 800 participants revealed 436 correlations between multiple SNPs in TRIM5 $\alpha$ and MS (Nexø et al., 2013). The significant 437 SNPs identified by Nexø and colleagues were located at the 5' end of the gene, near TRIM5 $\alpha$ 's 438 RING finger domain. The RING domain has been shown to be responsible for the protein's self439 ubiquitination activity (Yamauchi et al., 2008). Yamauchi and colleagues demonstrated through 440 proteasome inhibition that ubiquitination of TRIM5 $\alpha$ does not target it for degradation; instead, it 441 was shown to affect protein localisation, causing TRIM5 $\alpha$ to diffuse from localized cytoplasmic 442 bodies into the cytoplasm. It was later demonstrated that the RING domain of human TRIM5 $\alpha$ 443 also mediates the protein's K63 ubiquitination activity on TAK1 kinase; TAK1 ubiquitination 444 results in activation and induction of AP-1 and NF- $\mathrm{kB}$ in DCs (Pertel et al., 2011). The RING 445 domain thus appears to determine TRIM5a's inherent signalling capabilities, as well as 446 regulating the extent to which it interacts with viral capsids (as a consequence of protein 447 localization). As was previously described, TRIM5 $\alpha$-mediated downstream signalling has been 448 shown to be induced by interaction with retroviral capsids, so protein localization could 449 indirectly affect the protein's signal transduction properties (Pertel et al., 2011). Therefore, the 450 MS-associated SNP differences in the RING domain of TRIM5 $\alpha$ (Nexø et al., 2013) could alter 451 the protein's signalling activity in two ways; either by increasing its TAK1 ubiquitination 452 activity or by affecting its self-ubiquitination and localization, thus altering the frequency of 453 protein-capsid interactions. Overall, the current evidence suggests a potential mechanistic link 454 between TRIM5 $\alpha$ polymorphism, interaction with retroviral capsids, and dendritic cell 455 activation. 
457 We have seen that Fv-4, an ERV-encoded env gene in mice, maintains high levels of structural 458 similarity to its counterpart in exogenous $\mathrm{MuLV}$, and that this similarity allows the endogenous 459 retroviral element to interact with host proteins that recognize exogenous retroviruses. A similar 460 interaction could be occurring between HERV Gag proteins and TRIM5 $\alpha$. Due to their sequence 461 homology (Mueller-Lantzsch et. al, 2009), some HERV capsid elements (gag) likely overlap 462 with exogenous retroviruses in TRIM5 $\alpha$ binding specificity. In addition to TRIM5 $\alpha$ sequences, 463 recent findings have revealed that certain HERV-K SNPs also correlate with MS incidence 464 (Nexø et al., 2015), and that expression of some HERV-K gag genes is significantly increased in 465 PBMCs and brain cells extracted from MS patients (Laska et al., 2012; Bhetariya et al., 2017). 466 These HERV-K variants could comprise overactive regulatory sequences (LTRs) or gag proteins 467 with increased binding affinity for TRIM5 $\alpha$, though the observations of increased HERV-K 468 protein expression in MS (Laska et al., 2012; Bhetariya et al., 2017) suggest the former is more 469 relevant. Because TRIM5 $\alpha$ interaction with viral capsids increases the protein's downstream 470 signalling activity in DCs (Pertel et al., 2011), HERV gag variants with high avidity for the 471 restriction factor, or gag variants that are overexpressed, have the potential to increase 472 inflammatory cytokine production through TRIM5 $\alpha$-Gag interactions. I posit that the aberrant 473 activation of DCs in MS may arise due to the combination of TRIM5 $\alpha$ variants with increased 474 signal transduction properties and HERV-K variants that increase TRIM5 $\alpha$-mediated signalling. 475 Thus, the combination of rare SNPs at HERV and/or TRIM5 $\alpha$ loci could provide a genetic basis 476 for Multiple Sclerosis. A detailed mechanism of HERV and TRIM5 $\alpha$-mediated activation of DC 477 cells is shown in figure 4. 
479 To support this hypothesis, a more comprehensive genomic analysis is required to determine to 480 covariance of high-risk alleles at HERV-K and TRIM5 $\alpha$ loci in the incidence of MS. Future 481 research should also investigate the binding specificity of TRIM5 $\alpha$ for HERV-encoded Gag 482 proteins, and whether this interaction has any downstream effects in immune system cells, as 483 occurs with exogenous retroviral capsids. Despite the current lack of information, TRIM5 $\alpha$ and 484 HERV-K Gag interactions present an exciting new avenue for future investigation into the 485 molecular basis of MS pathogenesis.

486

487 Systemic Lupus Erythematosus. Systemic lupus erythematosus (SLE) is another 488 autoimmune disease that appears to have a significant relationship with HERVs. HERV 489 association with the disease has been established through observations of increased HERV 490 491 to HERV elements (Ogasawara et al., 2001; Hervé et al., 2001). SLE is a chronic inflammatory mRNA expression in afflicted patients, as well as the detection of circulating antibodies specific 492 autoimmune disease, characterized by an abnormal interferon response that causes an individual 493 to produce auto-reactive antibodies and immune complexes that target the body's own tissues 494 and organs (Maidhof and Hilas, 2012). One of the first pathological features of the disease to be detected was the presence of anti-dsDNA antibodies in the sera of affected patients; antibody concentration was observed to correlate with disease progression (Swaak et al., 1982). Type 1 497 activation of macrophages (a pro-inflammatory response) also appears to play a key role in 498 disease manifestation, as non-aberrant macrophage activation induces SLE remission in mice 499 (Schiffer et al., 2009; Li et al., 2015). 
501 The factors that induce pro-inflammatory activation of macrophages in SLE are not yet fully

502 understood, but dysregulation of the previously-mentioned AIM2 protein complex provides a

503 feasible mechanism. AIM2 is a foreign nucleic acid sensor, part of a group of proteins that

504 recognize so-called pathogen-associated molecular patterns (PAMPs) (Roers et al., 2016). The

505 AIM2 complex recognizes cytosolic DNA in a sequence-independent manner (Jin et al., 2012),

506 and upon recognition initiates an inflammatory response involving the maturation and release of

507 pro-inflammatory cytokines (Di Mocco et al., 2016). As macrophages commonly phagocytose

508 microbes and other pathogens, this DNA-sensing mechanism allows the cell to attune itself to an

509 active infection. Yet as well as pathogens, macrophages also engulf and digest apoptotic host

510 cells (Leers et al., 2002), and AIM2's sequence-independent recognition of DNA likely allows it

511 to activate upon binding apoptotic DNA. This has been observed in macrophages extracted from

512 SLE patients, in an experiment that also noted correlations between AIM2 expression and SLE

513 disease severity in humans (Zhang et al., 2013). Zhang and colleagues also designed an SLE

514 murine model, in which AIM2 knockdown significantly alleviated disease pathologies in

515 afflicted mice. Even more interestingly, their experiment showed that increased AIM2

516 expression in the mouse model correlated with increased anti-dsDNA antibody levels in serum, a

517 feature associated with human SLE pathogenesis (Swaak et al., 1982; Živković et al., 2014).

518 AIM2 has separately been implicated in the induction of MHC class II in cancer cells (Lee et al.,

519 2011), an antigen presentation complex expressed in macrophages (Harding and Geuze, 1992).

520 Aberrant AIM2 activity might therefore contribute to the production of anti-dsDNA antibodies

521 observed in SLE patients, through activation antigen presentation via MHC class II and induction

522 of a CD4+ T cell response against dsDNA antigens (Waisman et al., 1996; Underhill et al., 
523 1999). Regardless of its role in autoantibody formation, however, the evidence accrued by Zhang

524 and colleagues (2013) indicates an indispensable role for AIM2 in the pathogenesis of SLE.

525

526 As previously described, AIM2 operates (in part) under the control of MER41.AIM2, a single

527 HERV-derived promoter region. There is evidence that the etiology of SLE might relate to

528 epigenetic dysregulation of AIM2. A high-throughput analysis of CpG-containing promoters for

529 various genes revealed significant differences in AIM2 promoter methylation $(p=0.01)$ in

530 monozygotic twin pairs disconcordant for SLE (Javierre et al., 2010). Individuals with SLE

531 exhibited hypomethylation at the locus and corresponding increased expression of AIM2

532 compared with their healthy twin. While the analysis did not investigate the specific nature of

533 each promoter tested, MER41.AIM2 is known to be located in very close proximity to AIM2 and

534 has been demonstrated to be a STAT1 binding site, necessary for AIM2 expression under IFN- $\gamma$

535 stimulation (Chuong et al., 2016). It therefore serves as a probable candidate for epigenetic

536 modifications that affect $A I M 2$ expression. Given that $A I M 2$ 's activity appears to be intimately

537 linked with SLE pathogenesis (Zhang et al., 2013), the hypomethylation identified at the gene's

538 promoter by Javierre et al. (2009) provides a plausible mechanism for AIM2 overexpression and

539 suggests that MER41.AIM2 dysregulation might contribute to SLE pathogenesis, at least in some

540 patients. There remains much to be understood about the prevalence of co-opted ERV regulatory

541 sequences in the human genome and immune system, but given our current knowledge of ERV

542 co-option in other vertebrates, investigation into such elements presents promising opportunities

543 for future research. A more comprehensive analysis with larger sample sizes and more thorough

544 epigenetic profiling is necessary to elucidate the exact modifications that contribute to SLE.

545 Further research into the dysregulation of HERV promoter regions in SLE and other autoimmune 
546 diseases could reveal additional associations with disease incidence, given their intimate

547 relationship with the immune system.

548

549 The previously-detailed evidence suggests a meaningful role for AIM2 in SLE pathogenesis, but 550 Toll-like receptor 7 (TLR7) - another nucleic acid-sensing system - might also contribute to 551 disease progression through the detection of HERV-encoded products. TLR7 is an endosomal 552 ssRNA-binding receptor - expressed in immune system cells - that triggers a pro-inflammatory 553 response upon binding an ssRNA ligand (Brubaker et al., 2015). SLE patients exhibit increased 554 expression of TLR7 in peripheral blood mononuclear cells (PBMCs) (Guo et al., 2015). Guo and 555 colleagues also demonstrated that PBMCs from these patients were more sensitive to TLR7

556 stimulation by ssRNA. TLR7 has also been shown to be capable of recognizing ERV-derived 557 RNA in mice (Yu et al., 2012). ERVs have been implicated in the pathogenesis of murine SLE, 558 as early investigations revealed that the ERV-encoded Env protein gp70 was present in the sera 559 of multiple lupus-prone and healthy mouse strains, but that only sera from SLE-prone mice 560 contained anti-gp70 immune complexes (Izui et al., 1979). Mutant SLE mice with a TLR7 561 duplication exhibit increased levels of anti-ribonucleoprotein antibodies, including anti-gp70 562 complexes (Santiago-Raber et al., 2010). Santiago-Raber and colleagues also demonstrated that 563 B cell treatment with a TLR7 agonist significantly increased B cell activation in their murine 564 model. Other work has demonstrated that RNA-associated autoantigens activate autoantibody 565 production in SLE mouse B cells via combined activation of BCR and TLR7 (Lau et al., 2005). 566 PBMCs in humans with SLE have been shown to overexpress TLR7 (Guo et al., 2015), which 567 might increase the production of anti-RNA autoantibodies, as it does in mice (Santiago-Raber et 568 al., 2010). If TLR7 overstimulation does play a role in SLE pathogenesis, the effect is likely 
569 exacerbated by the increased concentrations of HERV mRNA - a TLR7 ligand - that are

570 associated with disease (Ogasawara et al., 2001).

571

572 Being derived from exogenous retroviruses, whose RNA and protein elements spontaneously

573 assemble, certain ERV elements may have the potential to produce complexes consisting of

574 immunogenic viral protein antigens associated with TLR7-stimulatory ssRNA. Such complexes

575 could be sufficient for co-stimulating BCR and TLR7 in B cells, even if the viral particles

576 themselves are not infectious. A model for this interaction is displayed in fig. 5. HERV virion-

577 RNA complexes have been detected in the sera of MS patients (Garson et al., 1998). Further

578 supporting this theory, anti-HERV-K Env antibodies have been detected in the sera of human

579 SLE patients (Hervé et al., 2001). However, in order to confirm the relevance of TLR7 and

580 HERV interactions in SLE, a more robust connection must first be established between specifc

581 molecular components and disease incidence. Genome-wide association studies could help to

582 identify TLR7 or HERV variantsthat are associated with disease. Epitopes from anti-HERV-K

583 Env antibodies in SLE patients could also be mapped to specific loci in the genome, which

584 would allow for more robust comparison between SLE-associated and healthy alleles at HERV-

$585 \mathrm{~K}$ sequences (assuming such variants exist). As was discovered in rheumatoid arthritis

586 (Freimanis et al., 2010), some of these epitopes might be cross-reactive with human proteins. In

587 vitro experiments could also test the ability of HERV virion-RNA complexes to stimulate TLR7

588 in B cells, assuming such complexes could be isolated or produced in situ. If a connection is

589 established between TLR7/HERV interaction and disease pathogenesis, the receptor presents a

590 suitable target for treatment via inhibition, due its functional redundancy with TLR8 (Yang et al., $5912005)$ 
592

\section{Conclusions}

594

595 The relationship between HERVs and autoimmune diseases has long been a controversial topic,

596 and a comprehensive model for their involvement in autoimmunity has not been synthesized.

597 Autoimmune diseases are particularly complex pathologies, usually not defined by specific

598 alleles or pathogens, but instead by the symptoms they produce and the complex interactions 599 observed in the immune systems of diseased patients. Therefore, it might be inaccurate to view

600 them as single diseases, but rather as sets of systemic effects whose contributing factors correlate 601 frequently. This uncertainty makes it difficult to make meaningful statements about AD etiology;

602 a diversity of factors could be capable of independently producing the same condition. The

603 purpose of this review is to summarize some of our knowledge with regards to HERV

604 involvement in immunity and autoimmunity, and to present some novel theories concerning the 605 mechanistic involvement of HERVs in autoimmune pathologies.

606

607 Co-opted ERV elements have been identified in animals and humans, but our current

608 understanding of human genomics cannot provide an accurate estimation of their biological

609 significance. ERV-encoded restriction factors have been identified in mice and other animals, but

610 human ERVs remain far less well-characterized. It has been observed that HERV activity

611 correlates with the incidence of multiple autoimmune diseases in humans, but specific

612 mechanistic hypotheses are required to determine their exact relationship. In this review, I argue

613 that HERV elements are not only capable of serving beneficial functions in their hosts; they may

614 also have to potential to trigger unwanted immune responses by producing immunostimulatory

615 viral antigens or acting as uncontrolled regulatory sequences. The domains of paleovirology and 
616 immunology will continue to overlap as more is discovered about present-day HERV activity in

617 the immune system. Investigation of endogenous viral elements is not only relevant to

618 evolutionary biology; it may also shed light on the nature of such autoimmune pathologies as

619 lupus and multiple sclerosis.

620

621

Acknowledgements

622

623 I would like to thank Dr. Michael Tristem for serving as my primary supervisor in the production 624 of this review. His insight and advice have been invaluable, and the project in its current form 625 would not exist without him.

626

627

628

\section{References}

629

630

631

632

633

634

635

636

637

638

639

640

641

642

643

644

645

646

647

648

649

650

651

652

653

654

655

656

657

Alcina, A., Abad-Grau, M., Fedetz, M., Izquierdo, G., Lucas, M., Fernández, Ó., Ndagire, D., Catalá-Rabasa, A., Ruiz, A., Gayán, J., Delgado, C., Arnal, C. \& Matesanz, F. (2011) Multiple Sclerosis Risk Variant HLA-DRB1*1501 Associates with High Expression of DRB1 Gene in Different Human Populations. Plos One. 7 (1), e29819.

Aletaha, D. \& Smolen, J. S. (2018) Diagnosis and management of rheumatoid arthritis: A review. Jama. 320 (13), 1360-1372.

Aletaha, D. \& Blüml, S. (2016) Therapeutic implications of autoantibodies in rheumatoid arthritis. RMD Open. 2 (1), e000009.

Aswad, A. \& Katzourakis, A. (2012) Paleovirology and virally derived immunity. Trends in Ecology \& Evolution. 27 (11), 627-636.

Balada, E., Ordi-Ros, J. \& Vilardell-Tarrés, M. (2009) Molecular mechanisms mediated by human endogenous retroviruses (HERVs) in autoimmunity. Reviews in Medical Virology. 19 (5), 273-286.

Balada, E., Vilardell-Tarrés, M. \& Ordi-Ros, J. (2010) Implication of Human Endogenous Retroviruses in the Development of Autoimmune Diseases. International Reviews of Immunology. 29 (4), 351-370.

Belshaw, R., Katzourakis, A., Paces, J., Burt, A. \& Tristem, M. (2005) High Copy Number in Human Endogenous Retrovirus Families is Associated with Copying Mechanisms in Addition to Reinfection. Molecular Biology and Evolution. 22 (4), 814-817.

Best, S., Tissier, P. L., Towers, G. \& Stoye, J. P. (1996) Positional cloning of the mouse retrovirus restriction gene Fvl. Nature. 382826. 
659 Bhardwaj, N., Maldarelli, F., Mellors, J. \& Coffin, J. M. (2014) HIV-1 infection leads to

660 increased transcription of human endogenous retrovirus HERV-K (HML-2) proviruses in vivo but not to increased virion production. Journal of Virology. 88 (19), 11108-11120.

662

663

664

665

Bhetariya, P. J., Kriesel, J. D. \& Fischer, K. F. (2017) Analysis of Human Endogenous Retrovirus Expression in Multiple Sclerosis Plaques. Journal of Emerging Diseases and Virology. 3 (2), 10.16966/2473-1846.133.

666

667

668

Bogdanos, D. P., Smyk, D. S., Rigopoulou, E. I., Mytilinaiou, M. G., Heneghan, M. A., Selmi, C. \& Eric Gershwin, M. (2012) Twin studies in autoimmune disease: Genetics, gender and environment. Journal of Autoimmunity; Special Issue: Gender, Sex Hormones, Pregnancy

670 and Autoimmunity. 38 (2), J156-J169.

671

672

673

Brubaker, S. W., Bonham, K. S., Zanoni, I. \& Kagan, J. C. (2015) Innate Immune Pattern

674

675

676

677

678

679

680

681

682

683

684

685

686

687

688

689

690

691

692

693

694

695

696

697

698

699

700

701

702

703

704

705

706

707

708

709

Recognition: A Cell Biological Perspective. Annual Review of Immunology. 33 257-290.

Choi, E. K., Gatenby, P. A., McGill, N. W., Bateman, J. F., Cole, W. G. \& York, J. R. (1988)

Autoantibodies to type II collagen: occurrence in rheumatoid arthritis, other arthritides, autoimmune connective tissue diseases, and chronic inflammatory syndromes. Annals of the Rheumatic Diseases. 47 (4), 313-322.

Chuong, E. B., Elde, N. C. \& Feschotte, C. (2016) Regulatory evolution of innate immunity through co-option of endogenous retroviruses. Science (New York, N.Y.). 351 (6277), 10831087.

Coffin JM, Hughes SH, Varmus HE, editors (1997) Retroviruses. Cold Spring Harbor (NY): Cold Spring Harbor Laboratory Press Immune Response to Retroviral Infection.

Daugherty, M. D. \& Malik, H. S. (2012) Rules of Engagement: Molecular Insights from HostVirus Arms Races. Annual Review of Genetics. 46 (1), 677-700.

Di Micco, A., Frera, G., Lugrin, J., Jamilloux, Y., Hsu, E., Tardivel, A., De Gassart, A., Zaffalon, L., Bujisic, B., Siegert, S., Quadroni, M., Broz, P., Henry, T., Hrycyna, C. A. \& Martinon, F. (2016) AIM2 inflammasome is activated by pharmacological disruption of nuclear envelope integrity. Proceedings of the National Academy of Sciences of the United States of America. 113 (32), E4671-E4680.

Ejtehadi, H. D., Freimanis, G. L., Ali, H. A., Bowman, S., Alavi, A., Axford, J., Callaghan, R. \& Nelson, P. N. (2006) The potential role of human endogenous retrovirus K10 in the pathogenesis of rheumatoid arthritis: a preliminary study. Annals of the Rheumatic Diseases. 65 (5), 612-616.

Emerman, M. \& Malik, H. S. (2010) Paleovirology - Modern Consequences of Ancient Viruses. PLoS Biology. 8 (2), e1000301.

Ercolini, A. M. \& Miller, S. D. (2009) The role of infections in autoimmune disease. Clinical and Experimental Immunology. 155 (1), 1-15.

Frank, J. A. \& Feschotte, C. (2017) Co-option of endogenous viral sequences for host cell function. Current Opinion in Virology; Animal Models for Viral Diseases • Paleovirology. 25 81-89. 
711 Franke, V., Ganesh, S., Karlic, R., Malik, R., Pasulka, J., Horvat, F., Kuzman, M., Fulka, H., 712 Cernohorska, M., Urbanova, J., Svobodova, E., Ma, J., Suzuki, Y., Aoki, F., Schultz, R. M., 713 Vlahovicek, K. \& Svoboda, P. (2017) Long terminal repeats power evolution of genes and 714 gene expression programs in mammalian oocytes and zygotes. Genome Research. 27 (8), 715 1384-1394.

716

717

718

719

720

721

722

723

724

725

726

727

728

729

730

731

732

733

734

735

736

737

738

739

740

741

742

743

744

745

746

747

748

749

750

751

752

753

754

755

756

757

758

759

760

761

762

763

Freimanis, G., Hooley, P., Ejtehadi, H. D., Ali, H. A., Veitch, A., Rylance, P. B., Alawi, A., Axford, J., Nevill, A., Murray, P. G. \& Nelson, P. N. (2010) A role for human endogenous retrovirus-K (HML-2) in rheumatoid arthritis: investigating mechanisms of pathogenesis. Clinical and Experimental Immunology. 160 (3), 340-347.

Garson, J., Tuke, P., Giraud, P., Paranhos-Baccala, G. \& Perron, H. (1998) Detection of virion-associated MSRV-RNA in serum of patients with multiple sclerosis. The Lancet. 351 (9095), 33.

Goff, S. P. (2014) Retrovirus Restriction Factors. Molecular Cell. 16 (6), 849-859.

Grigoriadis N. \& Pesch V. (2015) A basic overview of multiple sclerosis immunopathology. European Journal of Neurology. 22 3-13.

Guo, Q., Wang, Y., Xu, D., Nossent, J., Pavlos, N. J. \& Xu, J. (2018) Rheumatoid arthritis: pathological mechanisms and modern pharmacologic therapies. Bone Research. 6 (1), 15.

Guo, Y., Chai, Q., Zhao, Y., Li, P., Qiao, J. \& Huang, J. (2015) Increased activation of tolllike receptors-7 and -8 of peripheral blood mononuclear cells and upregulated serum cytokines in patients with pediatric systemic lupus erythematosus. International Journal of Clinical and Experimental Medicine. 8 (11), 20472-20480.

Harding, C. V. \& Geuze, H. J. (1992) Class II MHC molecules are present in macrophage lysosomes and phagolysosomes that function in the phagocytic processing of Listeria monocytogenes for presentation to T cells. The Journal of Cell Biology. 119 (3), 531-542.

Hervé, C. A., Lugli, E. B., Brand, A., Griffiths, D. J. \& Venables, P. J. (2001) Autoantibodies to human endogenous retrovirus- $K$ are frequently detected in health and disease and react with multiple epitopes. Clinical and Experimental Immunology. 128 (1), 75-82.

Honda, T. \& Tomonaga, K. (2016) Endogenous non-retroviral RNA virus elements evidence a novel type of antiviral immunity. Mobile Genetic Elements. 6 (3), e1165785.

Hummel, J., Kämmerer, U., Müller, N., Avota, E. \& Schneider-Schaulies, S. (2015) Human endogenous retrovirus envelope proteins target dendritic cells to suppress T-cell activation. European Journal of Immunology. 45 (6), 1748-1759.

Ikeda, H., Laigret, F., Martin, M. A. \& Repaske, R. (1985) Characterization of a molecularly cloned retroviral sequence associated with Fv-4 resistance. Journal of Virology. 55 (3), 768777.

International Human Genome Sequencing Consortium. (2001) Initial sequencing and analysis of the human genome. Nature. 409860.

Izui, S., McConahey, P. J., Theofilopoulos, A. N. \& Dixon, F. J. (1979) Association of circulating retroviral gp70-anti-gp70 immune complexes with murine systemic lupus erythematosus. The Journal of Experimental Medicine. 149 (5), 1099-1116. 
764

765

766

767

768

769

770

771

772

773

774

775

776

777

778

779

780

781

782

783

784

785

786

787

788

789

790

791

792

793

794

795

796

797

798

799

800

801

802

803

804

805

806

807

808

809

810

811

812

813

814

815

816

Javierre, B. M., Fernandez, A. F., Richter, J., Al-Shahrour, F., Martin-Subero, J., RodriguezUbreva, J., Berdasco, M., Fraga, M. F., O'Hanlon, T.,P., Rider, L. G., Jacinto, F. V., LopezLongo, F., Dopazo, J., Forn, M., Peinado, M. A., Carreño, L., Sawalha, A. H., Harley, J. B., Siebert, R., Esteller, M., Miller, F. W. \& Ballestar, E. (2009) Changes in the pattern of DNA methylation associate with twin discordance in systemic lupus erythematosus. Genome Research. 20 (2), 170-179.

Jin, T., Perry, A., Jiang, J., Smith, P., Curry, J. A., Unterholzner, L., Jiang, Z., Horvath, G., Rathinam, V., Johnstone, R. W., Hornung, V., Latz, E., Bowie, A. G., Fitzgerald, K. A. \& Xiao, T. S. (2012) Structures of The HIN Domain:DNA Complexes Reveal Ligand Binding and Activation Mechanisms of The AIM2 Inflammasome and IFI16 Receptor. Immunity. 36 (4), 561-571.

Katzourakis, A. \& Gifford, R. J. (2010) Endogenous Viral Elements in Animal Genomes. PLoS Genetics. 6 (11), e1001191.

Keiko, Y., Keiji, W., Kunikazu, T., Makoto, T. \& Tetsu, K. (2008) Ubiquitination of E3 ubiquitin ligase TRIM5a and its potential role. The FEBS Journal. 275 (7), 1540-1555.

Kim, J. W. \& Cunningham, J. M. (1993) N-linked glycosylation of the receptor for murine ecotropic retroviruses is altered in virus-infected cells. Journal of Biological Chemistry. 268 (22), 16316-16320.

Kozak, C. A. \& Chakraborti, A. (1996) Single Amino Acid Changes in the Murine Leukemia Virus Capsid Protein Gene Define the Target of Fv1 Resistance. Virology. 225 (2), 300-305.

Küçükali, C. İ., Kürtüncü, M., Çoban, A., Çebi, M. \& Tüzün, E. (2015) Epigenetics of Multiple Sclerosis: An Updated Review. Neuromolecular Medicine. 17 (2), 83-96.

Laska, M. J., Brudek, T., Nissen, K. K., Christensen, T., Møller-Larsen, A., Petersen, T. \& Nexø, B. A. (2012) Expression of HERV-Fc1, a human endogenous retrovirus, is increased in patients with active Multiple Sclerosis. Journal of Virology. 86 (7), 3713-3722.

Lau, C. M., Broughton, C., Tabor, A. S., Akira, S., Flavell, R. A., Mamula, M. J., Christensen, S. R., Shlomchik, M. J., Viglianti, G. A., Rifkin, I. R. \& Marshak-Rothstein, A. (2005) RNAassociated autoantigens activate $B$ cells by combined $B$ cell antigen receptor/Toll-like receptor 7 engagement. The Journal of Experimental Medicine. 202 (9), 1171-1177.

Lauring, A. S., Frydman, J. \& Andino, R. (2013) The role of mutational robustness in RNA virus evolution. Nature Reviews Microbiology. 11 (5), 327-336.

Lee, A., Nolan, A., Watson, J. \& Tristem, M. (2013) Identification of an ancient endogenous retrovirus, predating the divergence of the placental mammals. Philosophical Transactions of the Royal Society B: Biological Sciences. 368 (1626), 20120503.

Lee, J., Li, L., Gretz, N., Gebert, J. \& Dihlmann, S. (2011) Absent in Melanoma 2 (AIM2) is an important mediator of interferon-dependent and -independent HLA-DRA and HLA-DRB gene expression in colorectal cancers. Oncogene. 311242.

Leers, M. P. G., Björklund, V., Björklund, B., Jörnvall, H. \& Nap, M. (2002) An immunohistochemical study of the clearance of apoptotic cellular fragments. Cellular and Molecular Life Sciences CMLS. 59 (8), 1358-1365. 
818 Li, F., Yang, Y., Zhu, X., Huang, L. \& Xu, J. (2015) Macrophage Polarization Modulates

819 Development of Systemic Lupus Erythematosus. Cellular Physiology and Biochemistry. 37

820 (4), 1279-1288.

821

822

Limjoco, T. I., Dickie, P., Ikeda, H. \& Silver, J. (1993) Transgenic Fv-4 mice resistant to

823

824

825

826

827

828

829

830

831

832

833

834

835

836

837

838

839

840

841

842

843

844

845

846

847

848

849

850

851

852

853

854

855

856

857

858

859

860

861

862

863

864

865

866

867

868

Friend virus. Journal of Virology. 67 (7), 4163-4168.

Maidhof, W. \& Hilas, O. (2012) Lupus: An Overview of the Disease And Management Options. Pharmacy and Therapeutics. 37 (4), 240-249.

Mameli, G., Erre, G. L., Caggiu, E., Mura, S., Cossu, D., Bo, M., Cadoni, M. L., Piras, A., Mundula, N., Colombo, E., Buscetta, G., Passiu, G. \& Sechi, L. A. (2017) Identification of a HERV-K env surface peptide highly recognized in Rheumatoid Arthritis (RA) patients: a cross-sectional case-control study. Clinical and Experimental Immunology. 189 (1), 127131.

Mameli, G., Madeddu, G., Mei, A., Uleri, E., Poddighe, L., Delogu, L. G., Maida, I., Babudieri, S., Serra, C., Manetti, R., Mura, M. S. \& Dolei, A. (2013) Activation of MSRV-type endogenous retroviruses during infectious mononucleosis and Epstein-Barr virus latency: the missing link with multiple sclerosis? PloS One. 8 (11), e78474; e78474-e78474.

Mameli, G., Poddighe, L., Mei, A., Uleri, E., Sotgiu, S., Serra, C., Manetti, R. \& Dolei, A. (2012) Expression and Activation by Epstein Barr Virus of Human Endogenous RetrovirusesW in Blood Cells and Astrocytes: Inference for Multiple Sclerosis. Plos One. 7 (9), e44991.

Manghera, M. \& Douville, R. N. (2013) Endogenous retrovirus-K promoter: a landing strip for inflammatory transcription factors? Retrovirology. 10 16-16.

Masuda, M. \& Yoshikura, H. (1990) Construction and characterization of the recombinant Moloney murine leukemia viruses bearing the mouse Fv-4 env gene. Journal of Virology. 64 (3), 1033-1043.

Meda, F., Folci, M., Baccarelli, A. \& Selmi, C. (2010) The epigenetics of autoimmunity. Cellular and Molecular Immunology. 8 (3), 226-236.

Mi, S., Lee, X., Li, X., Veldman, G. M., Finnerty, H., Racie, L., LaVallie, E., Tang, X., Edouard, P., Howes, S., Keith Jr, J. C. \& McCoy, J. M. (2000) Syncytin is a captive retroviral envelope protein involved in human placental morphogenesis. Nature. 403785.

Morandi, E., Tanasescu, R., Tarlinton, R. E., Constantinescu, C. S., Zhang, W., Tench, C. \& Gran, B. (2017) The association between human endogenous retroviruses and multiple sclerosis: A systematic review and meta-analysis. Plos One. 12 (2), e0172415.

Mueller-Lantzsch, N., Sauter, M., Weiskircher, A., Kramer, K., Best, B., Buck, M. \& Grässer, F. (1993) Human Endogenous Retroviral Element K10 (HERV-K10) Encodes a Full-Length Gag Homologous 73-kDa Protein and a Functional Protease. AIDS Research and Human Retroviruses. 9 343-50.

Nelson, P. N., Roden, D., Nevill, A., Freimanis, G. L., Trela, M., Ejtehadi, H. D., Bowman, S., Axford, J., Veitch, A. M., Tugnet, N. \& Rylance, P. B. (2014) Rheumatoid Arthritis is

869 Pathogenic Mechanism of Disease? J Rheumatol. 41 (10), 1952. 
Nexø, B. A. (2018) A new theory on autoimmunity with reference to multiple

872

873

874

875

876

877

878

879

880

881

882

883

884

885

886

887

888

889

890

891

892

893

894

895

896

897

898

899

900

901

902

903

904

905

906

907

908

909

910

911

912

913

914

915

916

917

918

919

920

921

922

Nexø, B. A., Hansen, B., Nissen, K. K., Gundestrup, L., Terkelsen, T., Villesen, P., Bahrami, S., Petersen, T., Pedersen, F. S. \& Laska, M. J. (2013) Restriction Genes for Retroviruses Influence the Risk of Multiple Sclerosis. Plos One. 8 (9), e74063.

Nexø, B. A., Villesen, P., Nissen, K. K., Lindegaard, H. M., Rossing, P., Petersen, T., Tarnow, L., Hansen, B., Lorenzen, T., Hørslev-Petersen, K., Jensen, S. B., Bahrami, S., Lajer, M., Schmidt, K. L. M., Parving, H., Junker, P. \& Laska, M. J. (2015) Are human endogenous retroviruses triggers of autoimmune diseases? Unveiling associations of three diseases and viral loci. Immunologic Research. 64 55-63.

Ogasawara, H., Naito, T., Kaneko, H., Hishikawa, T., Sekigawa, I., Hashimoto, H., Kaneko, Y., Yamamoto, N., Maruyama, N. \& Yamamoto, N. (2001) Quantitative analyses of messenger RNA of human endogenous retrovirus in patients with systemic lupus erythematosus. J Rheumatol. 28 (3), 533.

Oja, M., Peltonen, J., Blomberg, J. \& Kaski, S. (2007) Methods for estimating human endogenous retrovirus activities from EST databases. BMC Bioinformatics. 8 S11-S11.

Pakpoor, J., Disanto, G., Gerber, J. E., Dobson, R., Meier, U. C., Giovannoni, G. \& Ramagopalan, S. V. (2013) The risk of developing multiple sclerosis in individuals seronegative for Epstein-Barr virus: a meta-analysis. Mult Scler. 19 (2), 162-166.

Patel, M. R., Emerman, M. \& Malik, H. S. (2011) Paleovirology: Ghosts and gifts of viruses past. Current Opinion in Virology. 1 (4), 304-309.

Perron, H., Jouvin-Marche, E., Michel, M., Ounanian-Paraz, A., Camelo, S., Dumon, A., Jolivet-Reynaud, C., Marcel, F., Souillet, Y., Borel, E., Gebuhrer, L., Santoro, L., Marcel, S., Seigneurin, J. M., Marche, P. N. \& Lafon, M. (2001) Multiple Sclerosis Retrovirus Particles and Recombinant Envelope Trigger an Abnormal Immune Response in Vitro, by Inducing Polyclonal VÎ216 T-Lymphocyte Activation. Virology. 287 (2), 321-332.

Pertel, T., Hausmann, S., Morger, D., Züger, S., Guerra, J., Lascano, J., Reinhard, C., Santoni, F., Uchil, P. D., Chatel, L., Bisiaux, A., Albert, M., Strambio-De-Castillia, C., Mothes, W., Pizzato, M., Grütter, M. \& Luban, J. (2011) TRIM5 is an innate immune sensor for the retrovirus capsid lattice. Nature. 472 (7343), 361-365.

Pincus, T., Hartley, J. W. \& Rowe, W. P. (1971) A major genetic locus affecting resistance to infection with Murine Leukemia Viruses. I. Tissue culture studies of naturally occurring viruses. The Journal of Experimental Medicine. 133 (6), 1219-1233.

Roers, A., Hiller, B. \& Hornung, V. (2016) Recognition of Endogenous Nucleic Acids by the Innate Immune System. Immunity. 44 (4), 739-754.

Santiago-Raber, M., Dunand-Sauthier, I., Wu, T., Li, Q., Uematsu, S., Akira, S., Reith, W., Mohan, C., Kotzin, B. L. \& Izui, S. (2010) Critical role of TLR7 in the acceleration of systemic lupus erythematosus in TLR9-deficient mice. Journal of Autoimmunity. 34 (4), 339-348.

Sastri, J. \& Campbell, E. M. (2011) Recent Insights into the Mechanism and Consequences of TRIM5a Retroviral Restriction. AIDS Research and Human Retroviruses. 27 (3), 231-238. 
923

924

925

926

927

928

929

930

931

932

933

934

935

936

937

938

939

940

941

942

943

944

945

946

947

948

949

950

951

952

953

954

955

956

957

958

959

960

961

962

963

964

965

966

967

968

969

970

971

972

973

974

975

Schiffer, L., Bethunaickan, R., Ramanujam, M., Huang, W., Schiffer, M., Tao, H., Madaio, M. M., Bottinger, E. P. \& Davidson, A. (2008) Activated Renal Macrophages Are Markers of Disease Onset and Disease Remission in Lupus Nephritis. Journal of Immunology. 180 (3), 1938-1947.

Seifarth, W., Frank, O., Zeilfelder, U., Spiess, B., Greenwood, A. D., Hehlmann, R. \& LeibLeib-Mösch, C. (2004) Comprehensive Analysis of Human Endogenous Retrovirus

Transcriptional Activity in Human Tissues with a Retrovirus-Specific Microarray. Journal of Virology. 79 (1), 341-352.

Sgarbanti, M., Remoli, A. L., Marsili, G., Ridolfi, B., Borsetti, A., Perrotti, E., Orsatti, R., Ilari, R., Sernicola, L., Stellacci, E., Ensoli, B. \& Battistini, A. (2008) IRF-1 Is Required for Full NF-KB Transcriptional Activity at the Human Immunodeficiency Virus Type 1 Long

Terminal Repeat Enhancer. Journal of Virology. 82 (7), 3632-3641.

Smith, D. A. \& Germolec, D. R. (1999) Introduction to immunology and autoimmunity. Environmental Health Perspectives. 107 Suppl 5 661-665.

Sutkowski, N., Conrad, B., Thorley-Lawson, D. \& Huber, B. T. (2001) Epstein-Barr Virus Transactivates the Human Endogenous Retrovirus HERV-K18 that Encodes a Superantigen. Immunity. 15 (4), 579-589.

Swaak, A. J., Groenwold, J., Aarden, L. A., Statius van Eps, L.W. \& Feltkamp, E. W. (1982) Prognostic value of anti-dsDNA in SLE. Annals of the Rheumatic Diseases. 41 (4), 388-395.

Taylor, G. M., Gao, Y. \& Sanders, D. A. (2001) Fv-4: Identification of the Defect in Env and the Mechanism of Resistance to Ecotropic Murine Leukemia Virus. Journal of Virology. 75 (22), 11244-11248.

Temin, H. M. (1981) Function of the retrovirus long terminal repeat. Cell. 28 (1), 3-5.

Underhill, D. M., Bassetti, M., Rudensky, A. \& Aderem, A. (1999) Dynamic interactions of macrophages with T cells during antigen presentation. The Journal of Experimental Medicine. 190 (12), 1909-1914.

Villesen, P., Aagaard, L., Wiuf, C. \& Pedersen, F. S. (2004) Identification of endogenous retroviral reading frames in the human genome. Retrovirology. 1 32-32.

Waisman, A., Zisman, E. \& Mozes, E. (1996) MHC Class II-Mediated T Cell Response to DNA. Cellular Immunology. 173 (1), 7-14.

Weiss, R. A. (2016) Human endogenous retroviruses: friend or foe? Apmis. 124 (1-2), 4-10.

Woo, J. L., Hyun, J. K., Hee, S. K. \& Kyung, L. J. (2003) Activation of the Human

Endogenous Retrovirus W Long Terminal Repeat by Herpes Simplex Virus Type 1 Immediate Early Protein 1. Molecules and Cells. 15 (1), 75-80.

Yan, Y., Buckler-White, A., Wollenberg, K. \& Kozak, C. A. (2008) Origin, antiviral function and evidence for positive selection of the gammaretrovirus restriction gene Fv1 in the genus Mus. Proceedings of the National Academy of Sciences of the United States of America. 106 (9), 3259-3263. 
976 Yang, K., Puel, A., Zhang, S., Eidenschenk, C., Ku, C., Casrouge, A., Picard, C., von

977 Bernuth, H., Senechal, B., Plancoulaine, S., Al-Hajjar, S., Al-Ghonaium, A., Maródi, L.,

978 Davidson, D., Speert, D., Roifman, C., Garty, B., Ozinsky, A., Barrat, F. J., Coffman, R. L.,

979 Miller, R. L., Li, X., Lebon, P., Rodriguez-Gallego, C., Chapel, H., Geissmann, F., Jouanguy,

980 E. \& Casanova, J. (2005) Human TLR-7-, -8-, and -9-Mediated Induction of IFN-a/ $\beta$ and $-\lambda$

981 Is IRAK-4 Dependent and Redundant for Protective Immunity to Viruses. Immunity. 23 (5),

982

983

984

985

986 465-478.

Yap, M. W., Nisole, S., Lynch, C. \& Stoye, J. P. (2004) Trim5a protein restricts both HIV-1 and murine leukemia virus. Proceedings of the National Academy of Sciences of the United States of America. 101 (29), 10786-10791.

987

988

Yu, P., Lübben, W., Slomka, H., Gebler, J., Konert, M., Cai, C., Neubrandt, L., Prazeres da Costa, O., Paul, S., Dehnert, S., Döhne, K., Thanisch, M., Storsberg, S., Wiegand, L., Kaufmann, A., Nain, M., Quintanilla-Martinez, L., Bettio, S., Schnierle, B., Kolesnikova, L., Becker, S., Schnare, M. \& Bauer, S. (2012) Nucleic Acid-Sensing Toll-like Receptors Are Essential for the Control of Endogenous Retrovirus Viremia and ERV-Induced Tumors. Immunity. 37 (5), 867-879.

994 Apoptotic DNA-induced Systemic Lupus Erythematosus via Arbitrating Macrophage Functional Maturation. Journal of Clinical Immunology. 33 (5), 925-937.

Živković, V., Stanković, A., Cvetković, T., Mitić, B., Kostić, S., Nedović, J. \& Stamenković, B. (2014) Anti-dsDNA, Anti-Nucleosome and Anti-C1q Antibodies as Disease Activity Markers in Patients with Systemic Lupus Erythematosus. Srpski Arhiv Za Celokupno Lekarstvo. 142

1003 431-6. 


\section{Figure 1}

Overview of the ERV co-option process with immune system functions annotated.

Retroviral endogenization occurs when an exogenous retrovirus integrates into the genome of a host germline cell. The integrated element can be co-opted to act as a coding gene or a promoter region for a host gene. Three functions of co-opted ERVs in the immune system that are discussed in this review are annotated. Recent evidence has also suggested that ERV RNA transcripts, in addition to ERV-encoded proteins and ERV-derived regulatory regions, may also mediate antiviral immunity in vertebrates.

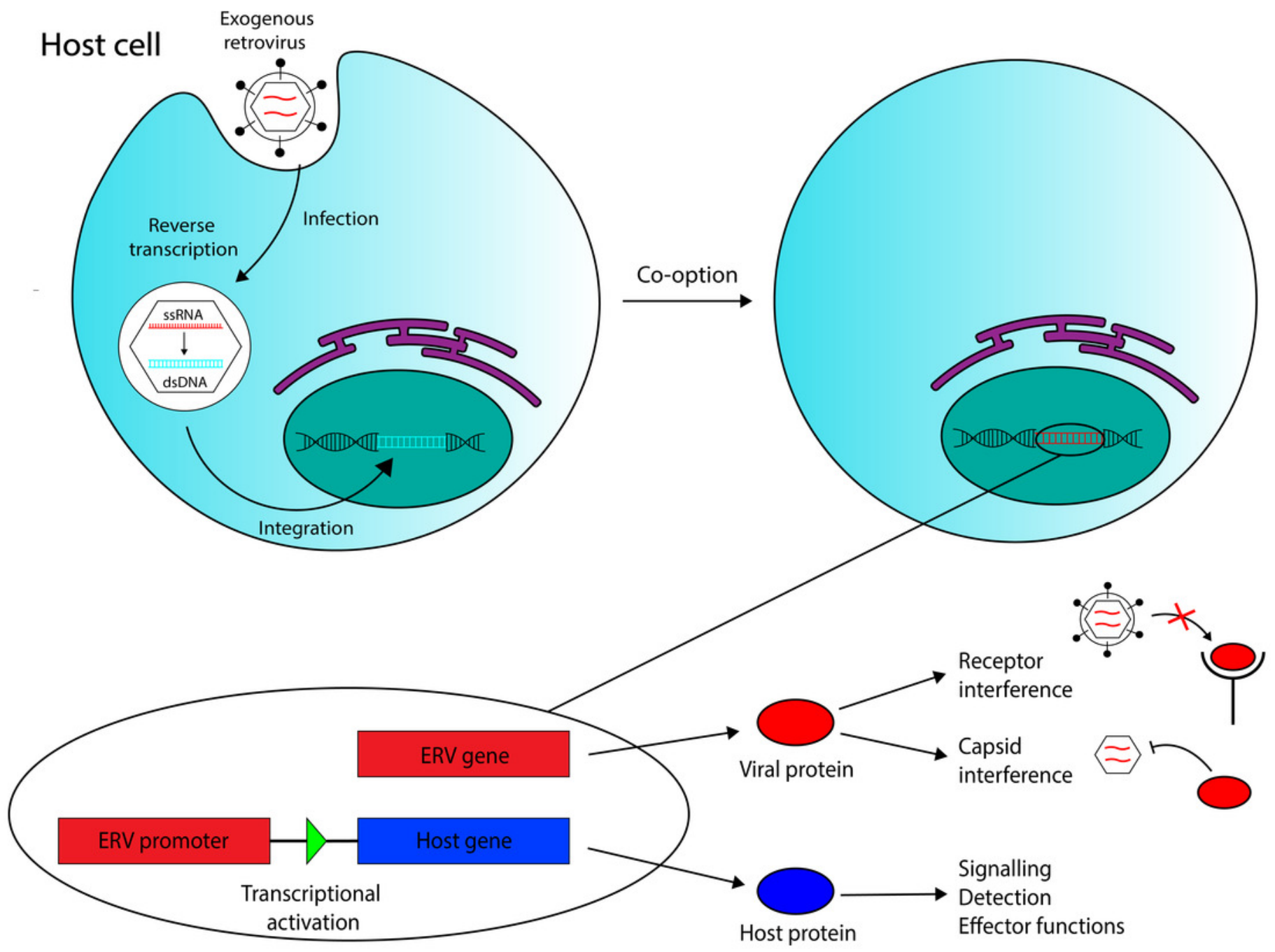


Figure 2

Hypothesized Fv-4 restriction mechanism in mice.

Fv-4 binds to MuLV receptors in the rough endoplasmic reticulum, and reduces their expression on the cell surface.

FV-4 reduces MuLV receptor expression on the cell surface

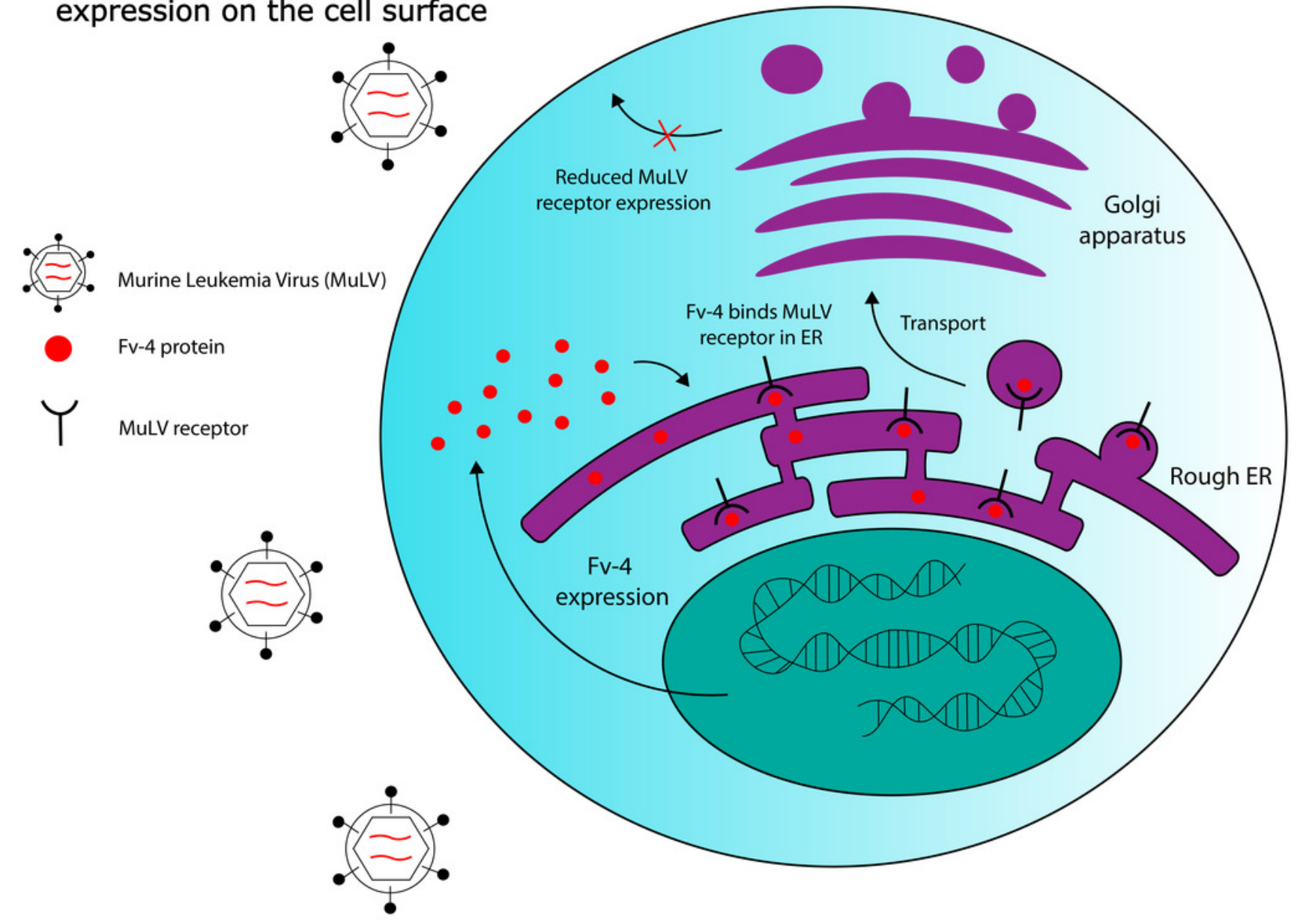




\section{Figure 3}

Effects of CRISPR/Cas knockout of a HERV regulatory element in the human immune system (adapted from Chuong et al., 2016).

Evidence obtained by Chuong et al. (2016) suggests that CRISPR/Cas knockout of MER41.AIM2 prevents expression of AIM2 upon treatment with IFN- $\gamma$ by inhibiting STAT1's binding to the promoter. These results imply a crucial role for the HERV element in cytokine signalling. (A) WT HeLa cells expressing AIM2 when exposed to IFN- $\gamma$. (B) MER41.AIM2-knockout HeLa cells that do not express AIM2 upon IFN- $\gamma$ stimulation.

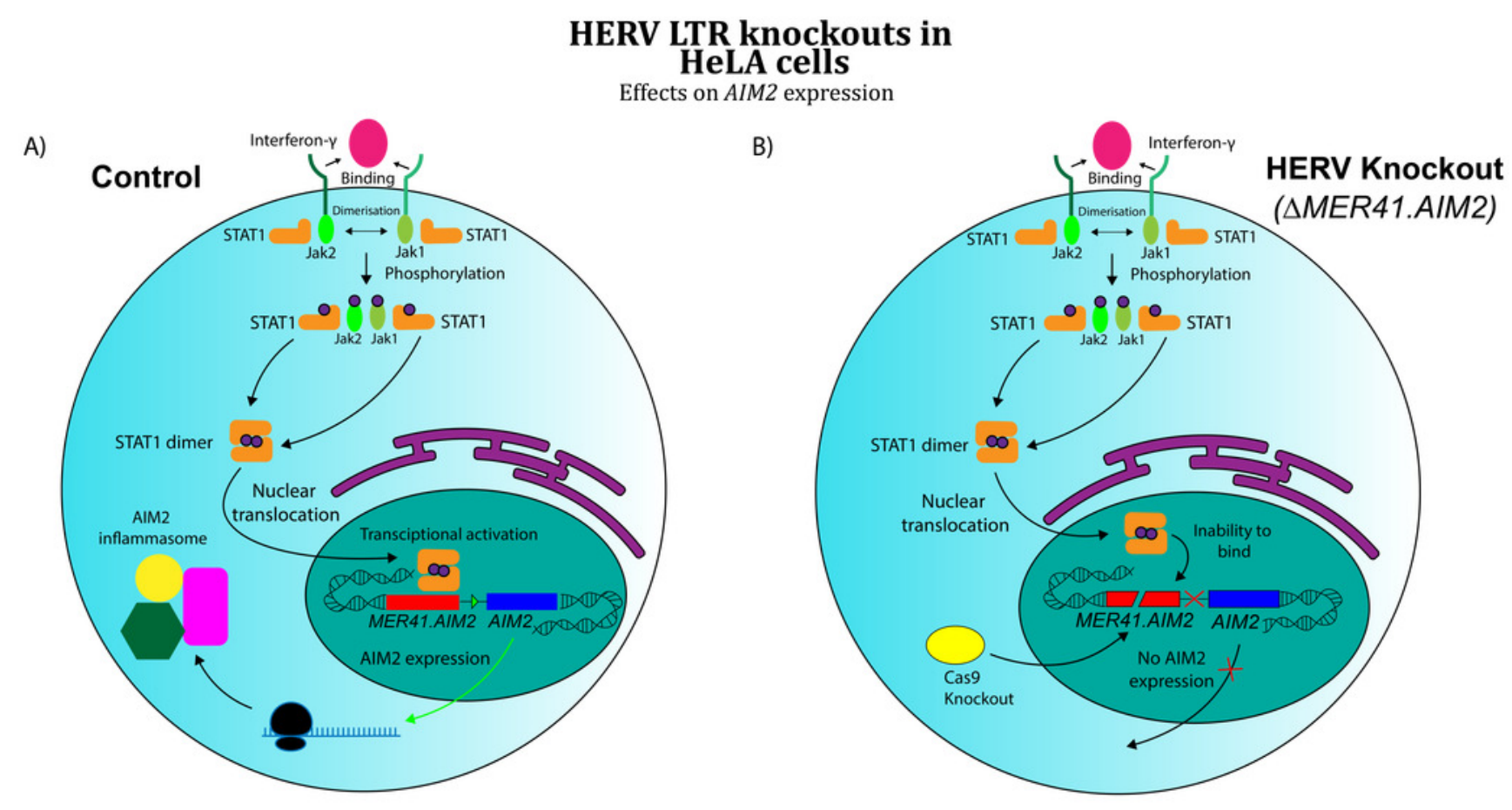


Figure 4

Potential role for HERVs in SLE autoantibody formation.

HERV virion-RNA complexes activate BCR and TLR7, leading to NF-KB activation through I-KB kinase (IKK) and stimulating the production of autoantibodies against HERV antigens.

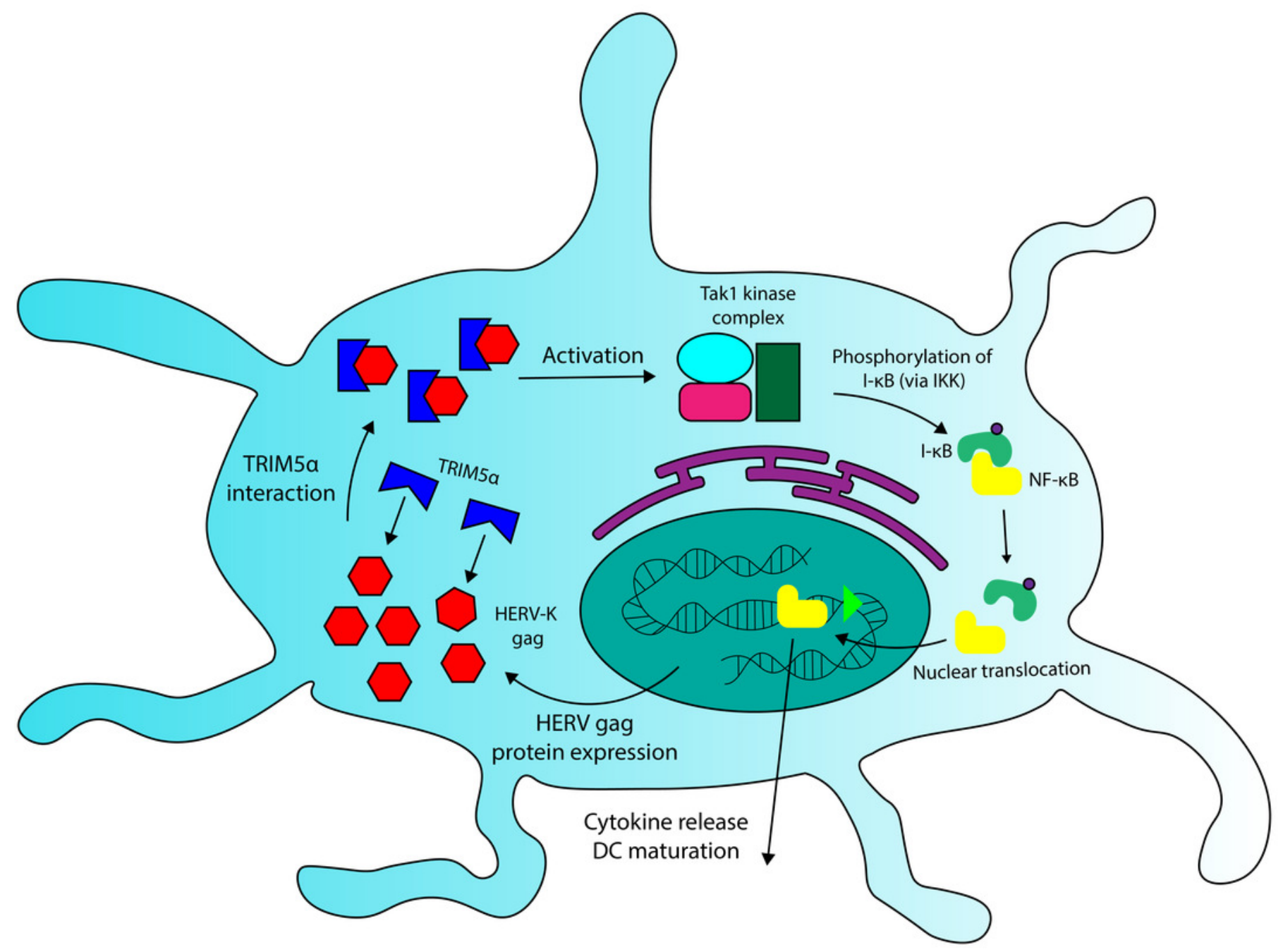




\section{Figure 5}

Potential role for HERVs in autoantibody production in SLE

There is evidence that HERV RNA-protein complexes formed in SLE patients could be capable of stimulating production of autoantibodies against HERV antigens. TLR7 stimulation by HERV RNA could contribute to this effect.

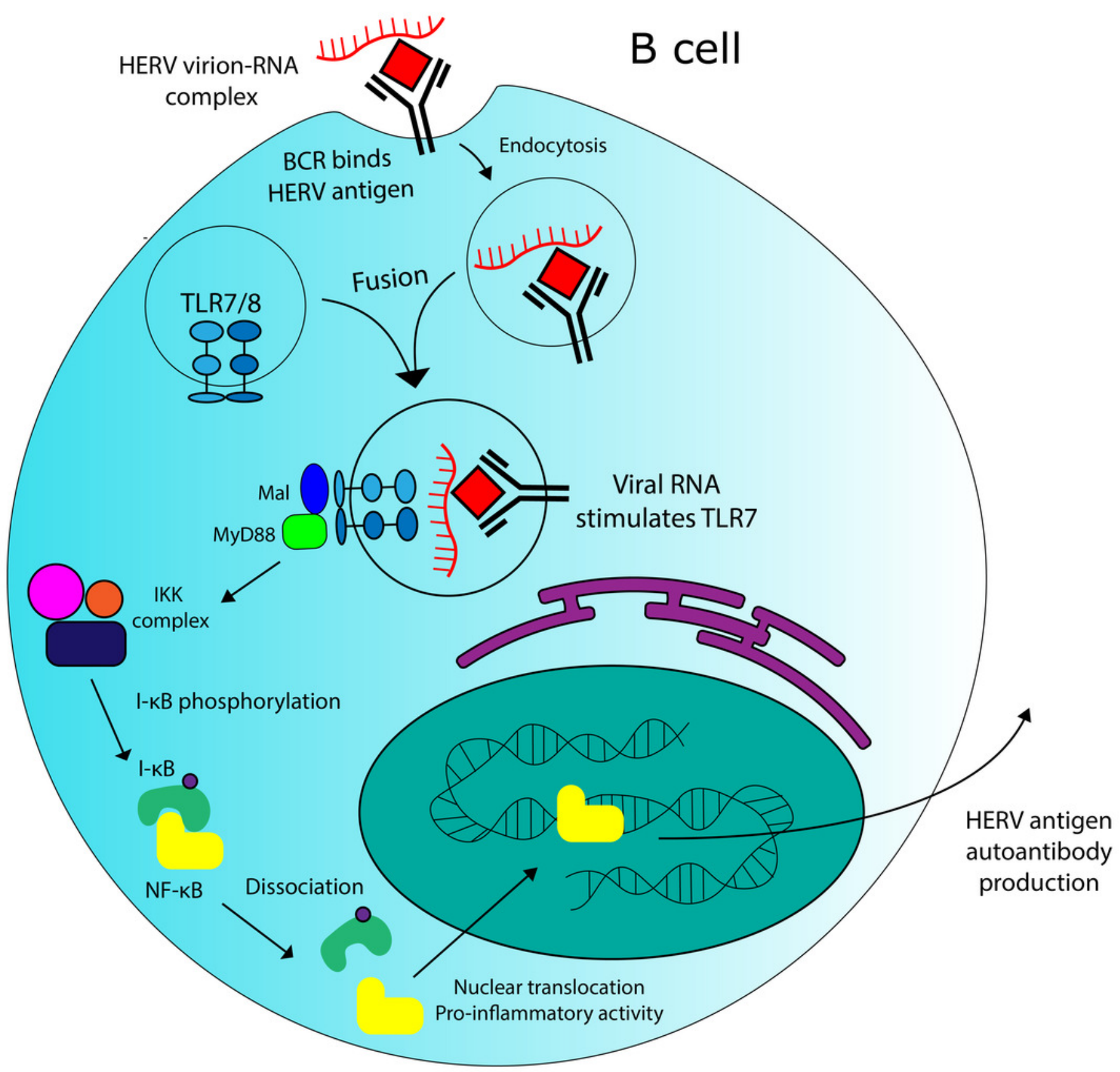

